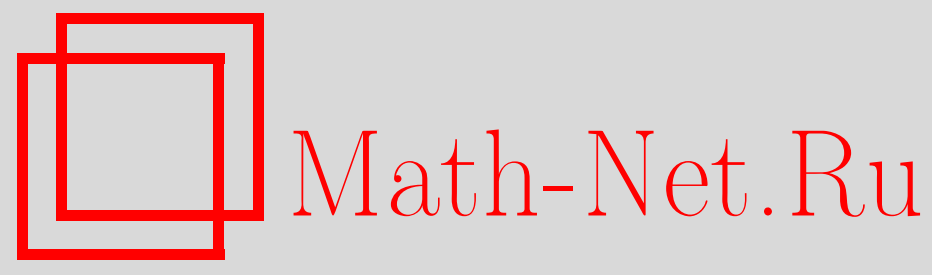

Д. В. Зайцев, О сложности сборки полных и полных двудольных графов, Дискрет. матем., 2008, том 20, выпуск 2, 82-99

DOI: https://doi.org/10.4213/dm1005

Использование Общероссийского математического портала Math-Net.Ru подразумевает, что вы прочитали и согласны с пользовательским соглашением http://www . mathnet.ru/rus/agreement

Параметры загрузки:

IP : 35.173 .219 .12

26 апреля 2023 г., 11:06:28 


\title{
О сложности сборки полных и полных двудольных графов
}

\author{
() 2008 г. Д. В. Зайцев
}

\begin{abstract}
Изучается сложность схем построения полных и двудольных полных графов с использованием двух операций склейки вершин, которые представляют собой отождествление пары вершин с удалением петель и кратных ребер. Первая операция применяется к парам вершин одного графа, вторая - к парам вершин двух графов, не имеющих общих элементов, в качестве исходного берется простейший граф, состоящий и пары вершин, соединенных ребром. Под сложностью построения понимается число применений операций над графами. Ранее были получены верхние оценки сложности построения полного и полного двудольного графа. В этой работе получены нижние оценки, что позволило получить порядок асимптотики построения этих графов.
\end{abstract}

\section{1. Введение}

В этой работе изучается сложность схем построения графов при помощи двух операций склейки вершин, которые заключаются в отождествлении пары вершин с удалением петель и кратных ребер. Первая операция применяется к паре вершин одного графа, вторая - к паре вершин двух графов, не имеющих общих элементов. В качестве простейшего берется граф, состоящий из пары вершин, соединенных ребром. Любой промежуточный результат, то есть построенный на каком-то этапе граф, разрешено использовать неоднократно. Под сложностью схемы понимается число применений операций над графами. Схемный подход к заданию графов рассматривался ранее, например, в [1], но вопрос о сложности схемы построения графов не изучался и использовались другие операции. Эта работа является продолжением исследований, начатых в [2], где были получены оценки функции Шеннона сложности схем для некоторых классов графов, а также верхние оценки сложности схемного задания для полного и полного двудольного графов. В этой работе получены нижние оценки, что позволило получить порядки сложности сборки полного и полного двудольного графов.

\section{2. Основные определения и результаты}

Пусть $K_{p, p}$ - полный двудольный граф с $p$ вершинами в каждой доле, $K_{n}-$ полный граф с $n$ вершинами. Пусть $V$ - класс неориентированных связных графов без петель и кратных ребер и $G \in V$. 
Определим две операции склейки графов. Двуместная операция склейки $O_{1}$ - отождествление двух заданных вершин пары графов, не имеющих общих элементов. Одноместная операция склейки $\mathrm{O}_{2}$ - отождествление двух заданных вершин одного графа. Всегда производится удаление петель и кратных ребер.

Сборкой графа $G$ назовем конечную последовательность графов $G_{1}, G_{2}, \ldots, G_{T}$, удовлетворяющую следующим условиям. Во-первых, $G_{T}$ изоморфен $G$, и $G_{1}$ состоит из пары вершин, соединенных ребром. Во-вторых, каждый граф $G_{s}, 1<s \leqslant T$, в последовательности получен в результате применения одной операции склейки следующим образом: одноместная операция применяется к графу изоморфному одному из $G_{1}, \ldots, G_{s-1}$, двуместная операция склейки применяется к паре графов, каждый из которых изоморфен одному из $G_{1}, \ldots, G_{s-1}$. Таким образом, каждый новый граф склеен из изоморфных предыдущим. В-третьих, требуется, чтобы каждый граф (кроме $G_{T}$ ) использовался, то есть участвовал в получении хотя бы одного графа с большим номером в данной последовательности.

Пусть длина последовательности сборки $G$ - число операций склейки, затраченных для построения последовательности, то есть $T-1$. Назовем последовательность сборки $G$ минимальной, если данная последовательность имеет наименьшую длину среди последовательностей сборки графа $G$. Сложностью $L(G)$ сборки $G$ назовем длину минимальной последовательности сборки $G$.

Теорема 1. При $p \rightarrow \infty$ справедливо соотношение $L\left(K_{p, p}\right) \asymp p$.

Теорема 2. При $p \rightarrow \infty$ справедливо соотношение $L\left(K_{n}\right) \asymp n$.

\section{3. Доказательство теоремы 1}

Пусть сеть сборки графа $G$, которую будем обозначать $\Sigma(G)$, - это ориентированный граф с двумя полюсами, в котором нет петель и разрешены кратные ребра. Каждой вершине сети взаимно однозначно соответствует некоторый граф из последовательности сборки. Если $G_{1}$ - первый граф в последовательности сборки, а $G_{T}$ последний, то первому полюсу сети $\Sigma(G)$ соответствует $G_{1}$, второму полюсу $-G_{T}$. Кроме того, каждой вершине сети, за исключением первого полюса, приписана одна операция из $\left\{O_{1}, O_{2}\right\}$ следующим образом. Пусть $\sigma$ - некоторая вершина сети $\Sigma(G)$, отличная от первого полюса. Пусть вершине $\sigma$ соответствует граф $G_{s}, 1<s \leqslant T$. Если $G_{s}$ в последовательности сборки получен применением операции $O_{1}$, то вершине $\sigma$ приписывается операция $O_{1}$, если применением $\mathrm{O}_{2}$, то приписывается $\mathrm{O}_{2}$. Вершины сети соединены ориентированными ребрами. Пусть $\varepsilon-$ некоторое ребро, входящее в $\sigma$, пусть $O_{k}, k \in\{1,2\},-$ операция, приписанная $\sigma$. Тогда началу $\varepsilon$ соответствует граф $G_{i}, 1 \leqslant i<s$, к копии которого (к изоморфному графу) применялась операция $O_{k}$ при получении $G_{s}$. Таким образом, степень захода вершины $\sigma$ равна двум, если ей приписана операция $O_{1}$, и равна единице, если ей приписана операция $O_{2}$. Степень исхода любой вершины сети, кроме второго полюса, должна быть больше нуля согласно третьему условию определения последовательности сборки. И, наконец, опишем последний пункт в определении $\Sigma(G)$. По определению последовательности сборки, каждый граф последовательности, кроме первого, получается в результате применения одной из операций склейки. Операции применяем так: выбираем (в зависимости от операции) один или пару графов последовательности, берем изоморфные им графы-копии, с этими копиями проводим склейку. Каждому ребру сети сопоставляем как раз копию графа, соответствующего началу данного ребра. Этот пункт определения имеет значение, когда в последовательности сборки рассматривается граф, 
полученный применением $O_{1}$ к паре копий одного графа. В сети сборки в этом случае возникает пара кратных ребер, которые нельзя отличить, если ссылаться на их концы. У этих ребер есть существенное отличие. Действительно, применяя операцию $O_{1}$ к копиям графа, соответствующего общему началу этих ребер, мы можем выбирать разные (не соответствующие друг другу при изоморфизме) вершины для склейки. Различать копии полезно, когда надо определить, какая вершина поглощается, а какая остается в результате склейки.

По определению для любой последовательности сборки графа $G$ можно построить сеть сборки. Поэтому, если мы получим нижнюю оценку числа вершин сети, справедливую для любой сети сборки $G$, то из нее мы получим нижнюю оценку числа операций в любой последовательности сборки графа $G$. Эта оценка верна и для последовательности сборки минимальной длины, следовательно, она будет оценкой сложности сборки $G$.

Назовем выделенным путем в сети сборки ориентированный путь, началом которого является первый полюс, соответствующий $G_{1}$, концом - второй полюс, соответствующий $G_{T}$. Заметим, что выделенный путь всегда является простой ориентированной цепью.

С этого момента будем ссылаться на графы, соответствующие вершинам выделенного пути, по их номеру в выделенном пути. Пусть $\tau$ - число вершин выделенного пути. Пусть $\widetilde{G}_{k}, 1 \leqslant k \leqslant \tau$, обозначает $k$-й по счету граф от начала выделенного пути. Началу выделенного пути в первом полюсе сети $\Sigma(G)$ соответствует $\widetilde{G}_{1} \cong G_{1}$, концу во втором полюсе $-\widetilde{G}_{\tau} \cong G_{T}$. Графы последовательности сборки, соответствующие графам выделенного пути, образуют подпоследовательность в последовательности сборки.

Определим подмножества вершин $A_{k}$ и $B_{k}, 1 \leqslant k \leqslant \tau$, в графе $\widetilde{G}_{k}$, принадлежащем некоторому выделенному пути в сборке графа $K_{p, p}$. Определяем их индукцией по направлению от конца к началу выделенного пути. Поскольку для произвольного выделенного пути в сборке $\Sigma\left(K_{p, p}\right)$ граф $\widetilde{G}_{\tau} \cong K_{p, p}$, основанием индукции является следующий факт: $A_{\tau}$ совпадает с одной долей $K_{p, p}, B_{\tau}$ совпадает с другой долей. Шаг индукции определяется так. Пусть $\widetilde{G}_{i}$ и $\widetilde{G}_{i+1}$ - два графа, которые принадлежат выделенному пути и соответствуют смежным вершинам сети, то есть, если применяется $O_{1}$, то $\widetilde{G}_{i+1}$ получен непосредственно из графа $G^{\prime}$, изоморфного $\widetilde{G}_{i}$ и из графа $G^{\prime \prime}$, который изоморфен некоторому графу, уже полученному в последовательности сборки. Если оба графа $G^{\prime}$ и $G^{\prime \prime}$ брались как копии $\widetilde{G}_{i}$, то считаем, что $\widetilde{G}_{i+1}$ получен из той копии $\widetilde{G}_{i}$, которой соответствует ребро выделенного пути. Если применяется $O_{2}$, то $\widetilde{G}_{i+1}$ получен непосредственно из графа $G^{\prime}$, изоморфного $\widetilde{G}_{i}$. Итак, по предположению индукции, уже определены подмножества $A_{i+1}$ и $B_{i+1}$ в множестве вершин графа $\widetilde{G}_{i+1}$. Определим $A_{i}$ и $B_{i}$ в множестве вершин графа $\widetilde{G}_{i}$. Рассмотрим произвольную вершину $v \in \widetilde{G}_{i}$. Возможны следующие случаи.

1. Вершина $v$ не участвует в операции склейки при переходе от $\widetilde{G}_{i}$ к $\widetilde{G}_{i+1}$, то есть вершина $v^{\prime} \in G^{\prime}$, соответствующая вершине $v$ (при рассмотрении графа $G^{\prime}$, изоморфного $\left.\widetilde{G}_{i}\right)$, не склеивается. Тогда $v \in A_{i}$, если $v^{\prime}$ попадает в подмножество $A_{i+1}$ в результате склейки, и $v \in B_{i}$, если $v^{\prime}$ попадает в подмножество $B_{i+1}$.

2. Вершина $v$ участвует в склейке с операцией $O_{1}$ при получении $\widetilde{G}_{i+1}$, то есть вершина $v^{\prime}$, соответствующая вершине $v$ при изоморфизме, склеилась с какой-то вершиной $v^{\prime \prime} \in G^{\prime \prime}$, где $G^{\prime \prime}$ изоморфен некоторому уже полученному ранее графу. Напомним, что выделенный путь проходит через ребро $G^{\prime}$. Тогда определяем, что $v \in A_{i}$, если $v^{\prime}$ после отождествления с $v^{\prime \prime}$ попадает в $A_{i+1}$, и $v \in B_{i}$, если $v^{\prime}$ после отождествления с $v^{\prime \prime}$ попадает в $B_{i+1}$.

3. Вершина $v$ участвует в склейке с операцией $O_{2}$ при получении $\widetilde{G}_{i+1}$, то есть вершина 
$v^{\prime} \in G^{\prime}$, соответствующая вершине $v$ при изоморфизме, склеилась с какой-то вершиной $w^{\prime} \in G^{\prime}$. Выделенный путь проходит через ребро, соответствующее $G^{\prime}$. Тогда определяем, что $v \in A_{i}$, если $v^{\prime}$ после отождествления с $w^{\prime}$ попадает в $A_{i+1}$, и $v \in B_{i}$, если $v^{\prime}$ после отождествления с $w^{\prime}$ попадает в $B_{i+1}$. Отметим, что $w \in \widetilde{G}_{i}$, соответствующая $w^{\prime} \in G^{\prime}$, принадлежит $A_{i}$, если $v \in A_{i}$, и принадлежит $B_{i}$, если $v \in B_{i}$.

Определение подмножеств вершин $A_{k}$ и $B_{k}$ закончено.

Рассмотрим пару простых следствий этой процедуры. Во-первых, множество вершин $\widetilde{G}_{k}$ есть $A_{k} \cup B_{k}$, во-вторых, $A_{k} \cap B_{k}=\varnothing$. Это можно установить по индукции. В графе $\widetilde{G}_{\tau}$ все вершины принадлежат или $A_{\tau}$, или $B_{\tau}$, и $A_{\tau} \cap B_{\tau}=\varnothing$, так как $\widetilde{G}_{\tau} \cong K_{p, p}$. Допустим, что множество вершин $\widetilde{G}_{i+1}$ есть $A_{i+1} \cup B_{i+1}$ и $A_{i+1} \cap B_{i+1}=\varnothing$. По определению при переходе от $\widetilde{G}_{i+1}$ к $\widetilde{G}_{i}$ каждую вершину $\widetilde{G}_{i}$ можно отнести к $A_{i}$ или $B_{i}$, но не к обоим одновременно. Таким образом, по индукции можно установить, что для любого $k$, $1 \leqslant k \leqslant \tau$, для определенных выше $A_{k}$ и $B_{k}$ все множество вершин $\widetilde{G}_{k}$ есть $A_{k} \cup B_{k}$ и $A_{k} \cap B_{k}=\varnothing$.

Пусть $\widetilde{G}_{i}$ и $\widetilde{G}_{j}, i \leqslant j$, - два графа, принадлежащие выделенному пути. Пусть $v_{i} \in \widetilde{G}_{i}$. Определим в графе $\widetilde{G}_{j}$ вершину $v_{j}$, потомка вершины $v_{i}$ для данного выделенного пути. Определять будем при помощи индукции, двигаясь по направлению от начала к концу выделенного пути. Будем считать, что $v_{i}$ сама является своим потомком. Пусть по предположению индукции определена вершина $v_{l} \in \widetilde{G}_{l}, i \leqslant l \leqslant j$ которая является потомком вершины $v_{i}$. Пусть $\widetilde{G}_{l} \cong G_{l}^{\prime}$, где $G_{l}^{\prime}$ приписан ребру выделенного пути. Вершина $v_{l}^{\prime} \in G_{l}^{\prime}$ соответствует $v_{l}$ при изоморфизме. Тогда $v_{l+1} \in \widetilde{G}_{l+1}-$ потомок $v_{i}$, если $\widetilde{G}_{l+1}$ принадлежит выделенному пути и выполнено одно из следующих условий:

(1) $v_{l}$ не участвует в склейке при переходе от $\widetilde{G}_{l}$ к $\widetilde{G}_{l+1}, v_{l+1}-$ вершина, в которую переходит $v_{l}^{\prime}$ после применения операции к $G_{l}^{\prime}$.

(2) $v_{l}$ участвует в склейке, $v_{l+1}$ получена в результате склейки $v_{l}^{\prime}$ с некоторой вершиной.

Определение потомка вершины $v_{i}$ закончено.

Заметим, что $v_{j} \in A_{j}$, если $v_{i} \in A_{i}$, и $v_{j} \in B_{j}$, если $v_{i} \in B_{i}$. Это верно, так как в противном случае существует шаг $l$, на котором потомок вершины из $A_{l}$ (из $B_{l}$ ) оказывается в $B_{l+1}\left(\right.$ в $\left.A_{l+1}\right)$, это противоречит определению $A_{l}\left(B_{l}\right)$. Заметим также, что в графе $\widetilde{G}_{i}$ может находиться несколько вершин помимо $v_{i}$, для которых $v_{j} \in \widetilde{G}_{j}$ является потомком. Это возможно, когда для некоторого $l, i \leqslant l<j$, при переходе от $\widetilde{G}_{l}$ к $\widetilde{G}_{l+1}$ применялась операция $O_{2}$. В этом случае две вершины из $\widetilde{G}_{l}$ переходят в одну вершину из $\widetilde{G}_{l+1}$ по ребру $G_{l}^{\prime}$ выделенного пути.

Для удобства, будем называть вершину $v_{i}$, для которой $v_{j}$ является потомком, родителем $v_{j}$. Будем говорить, что вершина (ребро) графа $\widetilde{G}_{l+1}$ из выделенного пути появляется на шаге $l+1$, если в предыдущем графе $\widetilde{G}_{l}$ выделенного пути нет родителя данной вершины (нет родителя конца данного ребра, смежного с родителем второго конца).

Рассмотрим $A_{j}$. Множество потомков вершин из множества $A_{i}$ образуют подмножество в $A_{j}$. Подмножество может совпадать со всем множеством. То же самое верно для $B_{i}$ и $B_{j}$. Обозначим через $A_{j}^{i}$ подмножество потомков вершин из $A_{i}$ в множестве $A_{j}$ и через $B_{j}^{i}$ подмножество потомков вершин $B_{i}$ в $B_{j}$.

Лемма 1. Для любой сети сборки $\Sigma\left(K_{p, p}\right)$, для любого вылеленного пути в сети сборки $\Sigma\left(K_{p, p}\right)$, для любой пары графов из данного выделенного пути $\widetilde{G}_{i} u \widetilde{G}_{j}$, где $1 \leqslant i<j \leqslant \tau$, для любой вершины $\omega_{j}$ такой, что $\omega_{j} \in A_{j} u \omega_{j} \notin A_{j}^{i}$, справедливо следующее: если $\omega_{j}$ смежна со всеми вершинами из $B_{j}^{i}$, то $j-i \geqslant\left|B_{i}\right|$. 
Доказательство. Рассмотрим произвольную сеть сборки графа $K_{p, p}$. В данной сети $\Sigma\left(K_{p, p}\right)$ рассмотрим произвольный выделенный путь. В этом выделенном пути рассмотрим пару графов $\widetilde{G}_{i}$ и $\widetilde{G}_{j}$. Для продолжения доказательства леммы докажем несколько предложений.

Предложение 1. В графе $\widetilde{G}_{i}$ не существует вериины-родителя $\omega_{i}$, для которой $\omega_{j}$ является потомком.

Доказательство. Поскольку по условию леммы $\omega_{j} \notin A_{j}^{i}$, в графе $\widetilde{G}_{i}$ не существует вершины-родителя $\omega_{i}$, для которой $\omega_{j}$ является потомком. Если бы такая вершина $\omega_{i}$ существовала в $\widetilde{G}_{i}$, то она принадлежала бы или $A_{i}$, или $B_{i}$ (см. следствия определения этих подмножеств). Если она принадлежит $A_{i}$, то получаем противоречие так: у любой вершины из $A_{i}$, в частности, у $\omega_{i}$ есть единственный потомок в $\widetilde{G}_{j}$ (по определению вершины-потомка). Этот потомок принадлежит $A_{j}^{i}$ (по определению $A_{j}^{i}$ ), но $\omega_{j} \notin A_{j}^{i}$ по условию леммы 1 . Если $\omega_{j}$ является потомком вершины из $B_{i}$, то она должна принадлежать $B_{j}$ (см. замечание к определению вершины-потомка), что также противоречит условию леммы 1. Предложение 1 доказано.

Следствие 1. Существует минимальное $l, i<l \leqslant j$, такое, что в графе выделенного пути $\widetilde{G}_{l}$ существует вершина $\omega_{l}$ такая, что $\omega_{l} \in A_{l}, \omega_{l} \notin A_{l}^{i}$, для которой $\omega_{j}$ является потомком.

Для каждого $k, l \leqslant k \leqslant j$, определим подмножество $R_{k}$ вершин графа $\widetilde{G}_{k}$. Для каждой вершины из $R_{k}$ вершина $\omega_{j}$ является потомком. Вершины $R_{k}$ не являются потомками вершин из $\widetilde{G}_{k-1}$. Заметим, что $R_{k}$ может быть пусто, но по следствию 1 существует $k$ такое, что $R_{k}$ не пусто. Заметим также, что $R_{k} \subseteq A_{k} \backslash A_{k}^{i}$.

Предложение 2. Для любого $k, l \leqslant k \leqslant j$, если подмножество $R_{k}$ не пусто, то $\widetilde{G}_{k}$ был получен в результате применения операџии $O_{1}$.

Доказательство. Пусть $\omega_{k} \in R_{k}$. Если $\widetilde{G}_{k}-$ результат применения операции $O_{2}$, то все его вершины являются потомками вершин из $\widetilde{G}_{k-1}$ и $\omega_{k}$ является потомком некоторой вершины $\omega_{k-1} \in \widetilde{G}_{k-1}$, но это противоречит определению $R_{k}$. Предложение 2 доказано.

Будем говорить, что два непересекающихся подмножества вершин графа связаны ребром, если в графе существует хотя бы одно ребро, один конец которого принадлежит первому подмножеству, второй - второму подмножеству.

Рассмотрим графы $\widetilde{G}_{k}$ и $\widetilde{G}_{k+1}, i \leqslant k<j$. Пусть $\widetilde{G}_{k}^{\prime}$ соответствует ребру с концами $\widetilde{G}_{k}$ и $\widetilde{G}_{k+1}$, лежащему в выделенном пути сети $\Sigma\left(K_{p, p}\right)$.

Предложение 3. Для любого $k, l-1 \leqslant k \leqslant j-1$, справедливо следующее утверждение. Если $R_{k+1}$ и $B_{k+1}^{i}$ связаны ребром, то

(a) при получении $\widetilde{G}_{k+1}$ применялась $O_{1}$,

(б) одна из двух вершин для склейки бралась из $B_{k}^{i}$,

(в) не более одной вершины из $B_{k+1}^{i}$ связано ребром с подмножеством $R_{k+1}$. 


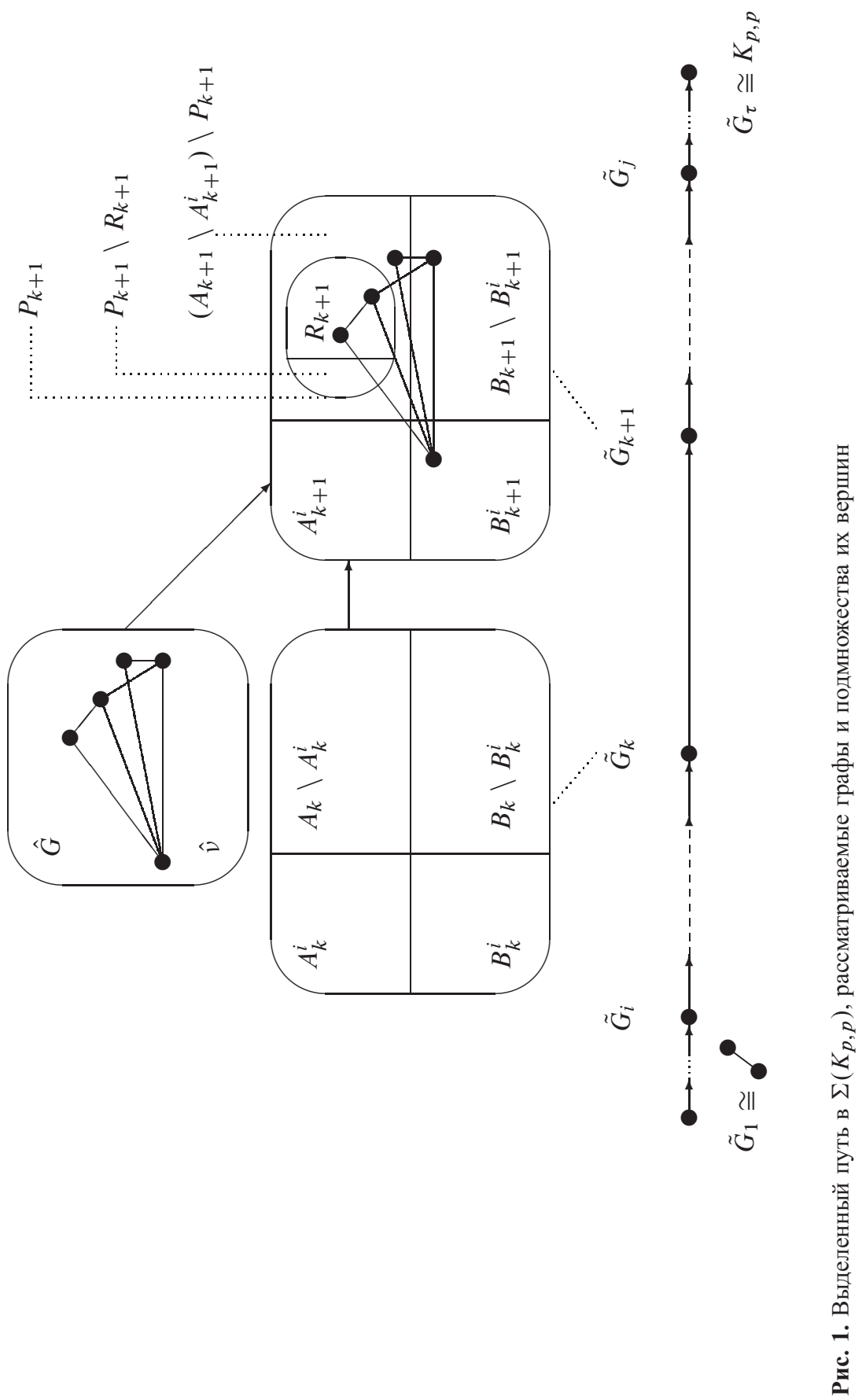


Доказательство. Утверждение (а) следует из предложения 2.

Докажем утверждение (б). Пусть $\widehat{G}$ - граф, который был склеен операцией $O_{1}$ с $\widetilde{G}_{k}^{\prime}$ для получения $\widetilde{G}_{k+1}$. Пусть $\hat{v} \in \widehat{G}-$ вершина, выбранная в $\widehat{G}$ для склейки. Рассмотрим следующие два факта. Во-первых, любая вершина в $R_{k+1}$ перешла из $\widehat{G}$ (без склейки), так как по определению в $R_{k+1}$ нет потомков $\widetilde{G}_{k}$. Во-вторых, $B_{k+1}^{i}$ содержит только потомков вершин из $B_{i}$ по определению. Из этих двух фактов следует, что склейка $\hat{v}$ с любой вершиной из $A_{k} \cup B_{k} \backslash B_{k}^{i}$ не может привести к связи ребром $R_{k+1}$ и $B_{k+1}^{i}$. Первый факт говорит о том, что смежные с $B_{k}^{i}$ вершины не могли перейти в $R_{k+1}$ из $\widetilde{G}_{k}$ при переходе к $\widetilde{G}_{k+1}$ (ни путем склейки, ни без склейки). Второй факт говорит о том, что в $B_{k+1}^{i}$ не могла попасть смежная с $R_{k+1}$ вершина, перейдя из $\widehat{G}$ без склейки с вершиной из $B_{k}^{i}$. Следовательно, существует вершина из $B_{k}^{i}$, которая клеится с вершиной из $\widehat{G}$.

Докажем утверждение (в). Ребра, связывающие $R_{k+1}$ с $B_{k+1}^{i}$, согласно пункту (б) могут появиться только в результате склейки некоторой вершины $\hat{v} \in \hat{G}$ с вершиной из $B_{k}^{i}$. За одну операцию можно склеить только одну вершину из $B_{k}^{i}$ с вершиной $\hat{v}$. Предложение 3 доказано.

Пусть $P_{k}$ - подмножество родителей $\omega_{j}$ в $\widetilde{G}_{k}$. Заметим, что $P_{k} \subseteq A_{k} \backslash A_{k}^{i}, R_{k} \subseteq P_{k}$ и что вершины $P_{k} \backslash R_{k}$ могут иметь родителей в $\widetilde{G}_{k-1}$. В графе $\widetilde{G}_{k}$ (и соответственно в $\widetilde{G}_{k}^{\prime}$, изоморфном ему) выделим следующие пять подмножеств вершин: $P_{k},\left(A_{k} \backslash A_{k}^{i}\right) \backslash P_{k}$, $A_{k}^{i}, B_{k} \backslash B_{k}^{i}, B_{k}^{i}$. Очевидно, что они не пересекаются, и их объединение равно множеству вершин $\widetilde{G}_{k}$.

Определим функцию $\varphi(k)$ как число вершин в $B_{k}^{i}$, которые не смежны ни с одной вершиной из $P_{k}$.

Рассмотрим возможности применения операции $O_{1}$ к графу $\widetilde{G}_{k}^{\prime}$. Одна вершина из отождествляемой пары должна принадлежать одному из пяти перечисленных выше подмножеств $P_{k},\left(A_{k} \backslash A_{k}^{i}\right) \backslash P_{k}, A_{k}^{i}, B_{k} \backslash B_{k}^{i}, B_{k}^{i}$. Следовательно, есть 5 возможностей выбора подмножества, путем указания вершины из $\widetilde{G}_{k}^{\prime}$ для склейки $O_{1}$.

Предложение 4. Для любого $k, i \leqslant k<j$, если при получении $\widetilde{G}_{k+1}$ в выделенном пути применяется операция $O_{1}$, то $0 \leqslant \varphi(k)-\varphi(k+1) \leqslant 1$, причем равенство единице возможно только тогда, когда склеиваемая вериина из $\widetilde{G}_{k}^{\prime}$ принадлежит $B_{k}^{i}$.

Доказательство. Пусть $u_{k} \in \widetilde{G}_{k}^{\prime}, i \leqslant k<j$, и $\hat{v} \in \widehat{G}$ - пара вершин, к которым применяется $O_{1}$. Рассмотрим все возможности выбора пары вершин для склейки. Отметим, что при применении $O_{1}$ нас интересуют только возможности выбора вершины из $\widetilde{G}_{k}^{\prime}$. Второй граф $\widehat{G}$, участвующий в склейке, не принадлежит выделенному пути, следовательно, $\widehat{G}$ не имеет своих подмножеств, определяемых через выделенный путь. Поэтому в каждом рассматриваемом случае из $\widehat{G}$ берется произвольная вершина. Возможны 5 случаев.

(1) $u_{k} \in A_{k}^{i}$.

(2) $u_{k} \in P_{k}$.

(3) $u_{k} \in\left(A_{k} \backslash A_{k}^{i}\right) \backslash P_{k}$.

(4) $u_{k} \in B_{k} \backslash B_{k}^{i}$.

Для склеек вида 1-4 верно равенство $\varphi(k)=\varphi(k+1)$. Действительно, в этих склейках не участвуют вершины из $B_{k}^{i}$, значит, не может измениться их смежность с другими 
вершинами. Число вершин также не меняется (в частности, не увеличивается, так как все они - потомки $\left.B_{i}\right)$, то есть в этих случаях $\left|B_{k}^{i}\right|-\left|B_{k+1}^{i}\right|=0$.

(5) $u_{k} \in B_{k}^{i}$.

Если в результате применения операции $O_{1}$ в графе $\widetilde{G}_{k+1}$ содержатся вершины, у которых нет родителей в $\widetilde{G}_{k}$, то эти вершины содержатся в $A_{k+1} \backslash A_{k+1}^{i}$ или в $B_{k+1} \backslash B_{k+1}^{i}$. Подмножество таких вершин, для которых $\omega_{j}$ является потомком, мы обозначили $R_{k+1}$. По предложению 3 не более одной вершины из $B_{k+1}^{i}$ связано ребрами с $R_{k+1} \subset P_{k+1}$. Следовательно, $\varphi(k)-\varphi(k+1) \leqslant 1$. При операции $O_{1}$ справедливо равенство $\left|B_{k}^{i}\right|-\left|B_{k+1}^{i}\right|=0$, и число вершин, смежных с $P_{k}$, не может уменьшиться, значит, $0 \leqslant \varphi(k)-\varphi(k+1)$. Если $R_{k+1}=\varnothing$ после применения операции $O_{1}$, то не меняется смежность вершин из $B_{k}^{i}$, и также $\left|B_{k}^{i}\right|-\left|B_{k+1}^{i}\right|=0$, значит, $\varphi(k)=\varphi(k+1)$.

Получаем, что только в случае 5 разность $\varphi(k)-\varphi(k+1)$ может быть равной 1 . Предложение 4 доказано.

Рассмотрим возможности применения операции $O_{2}$ к графу $\widetilde{G}_{k}^{\prime}$ с точки зрения выбора пары вершин для склейки. Каждая вершина из отождествляемой пары может принадлежать одному из пяти перечисленных выше подмножеств. Пары отождествляемых вершин не упорядочены, обе вершины в паре могут принадлежать одному подмножеству. Следовательно, есть 15 возможностей выбора пары подмножеств указанием пары вершин для склейки $\mathrm{O}_{2}$.

Предложение 5. Для любого $k, i \leqslant k<j$, если при получении $\widetilde{G}_{k+1}$ в выделенном пути применяется операция $\mathrm{O}_{2}$, то $0 \leqslant \varphi(k)-\varphi(k+1) \leqslant 1$, причем равенство единице возможно только тогда, когда обе склеиваемые вершины принадлежат $B_{k}^{i}$, или одна принадлежит $B_{k}^{i}$, вторая $-B_{k} \backslash B_{k}^{i}$.

Доказательство. Пусть $u_{k}$ и $v_{k}$ - пара вершин графа $\widetilde{G}_{k}^{\prime}, i \leqslant k<j$, к которым применяется $\mathrm{O}_{2}$.

Рассмотрим все возможности выбора пары вершин для склейки.

(1) $u_{k} \in P_{k}, v_{k} \in B_{k} \backslash B_{k}^{i}$.

(2) $u_{k} \in P_{k}, v_{k} \in B_{k}^{i}$.

(3) $u_{k} \in\left(A_{k} \backslash A_{k}^{i}\right) \backslash P_{k}$ и $v_{k} \in B_{k} \backslash B_{k}^{i}$.

(4) $u_{k} \in B_{k}^{i}$ и $v_{k} \in\left(A_{k} \backslash A_{k}^{i}\right) \backslash P_{k}$.

(5) $u_{k} \in B_{k} \backslash B_{k}^{i}$ и $v_{k} \in A_{k}^{i}$.

(6) $u_{k} \in B_{k}^{i}$ и $v_{k} \in A_{k}^{i}$.

Склеек вида 1-6 нет по определению $A_{k}$ и $B_{k}$.

(7) $u_{k} \in P_{k}, v_{k} \in A_{k}^{i}$.

(8) $u_{k} \in P_{k}, v_{k} \in\left(A_{k} \backslash A_{k}^{i}\right) \backslash P_{k}$.

Склеек вида 7 и 8 нет. Действительно, $u_{k} \in P_{k}$ только тогда, когда результат склейки $u_{k}$ и $v_{k}$ является родителем $\omega_{j}$ в $\widetilde{G}_{k+1}$. При выполнении этого условия вершина $v_{k}$ также обязана принадлежать $P_{k}$, так как она благодаря данной склейке также является родителем $\omega_{j}$. Однако последнее не выполняется в случаях 7 и 8. 
(9) $u_{k} \in A_{k}^{i}, v_{k} \in\left(A_{k} \backslash A_{k}^{i}\right) \backslash P_{k}$.

(10) $u_{k}$ и $v_{k}$ принадлежат $A_{k}^{i}$.

(11) $u_{k}$ и $v_{k}$ принадлежат $\left(A_{k} \backslash A_{k}^{i}\right) \backslash P_{k}$.

(12) $u_{k}$ и $v_{k}$ принадлежат $P_{k}$.

Заметим, что в этом случае $\left|P_{k}\right|=\left|P_{k+1}\right|+1$; степень потомка $u_{k}$ и $v_{k}$ может быть не больше суммы степеней $u_{k}$ и $v_{k}$.

(13) $u_{k}$ и $v_{k}$ принадлежат $B_{k} \backslash B_{k}^{i}$.

Для склеек вида 9-13 справедливо равенство $\varphi(k)=\varphi(k+1)$. Это верно, так как в этих склейках не участвуют вершины из $B_{k}^{i}$, значит, не может измениться ни число вершин в $B_{k}^{i}$, ни их смежность с другими вершинами.

(14) $u_{k} \in B_{k}^{i}$ и $v_{k} \in B_{k} \backslash B_{k}^{i}$.

Для операции такого вида $0 \leqslant \varphi(k)-\varphi(k+1) \leqslant 1$. Равенство единице возможно, когда $v_{k}$ смежна с одной или с несколькими вершинами из $P_{k}$. Больше единицы эта разность быть не может потому, что за одну операцию склейки только одна вершина $u_{k} \in B_{k}^{i}$ могла отождествиться с некоторой вершиной из $B_{k} \backslash B_{k}^{i}$.

(15) $u_{k} \in B_{k}^{i}$ и $v_{k} \in B_{k}^{i}$.

Для операции такого вида $0 \leqslant \varphi(k)-\varphi(k+1) \leqslant 1$. Равенство единице возможно, когда хотя бы одна вершина $\left(u_{k}\right.$ или $\left.v_{k}\right)$ не имеет смежной вершины из $P_{k}$. Больше единицы эта разность быть не может потому, что в результате отождествления пары вершин, исчезает не более одной вершины.

Таким образом, получаем, что только в случаях 14 и $15 \varphi(k)-\varphi(k+1)$ может быть равной 1 .

Предложение 5 доказано.

Итак, при рассмотрении пяти подмножеств вершин некоторого $\widetilde{G}_{k}, i \leqslant k<j$, возникло 5 способов применения $O_{1}$ и 15 способов применения $O_{2}$ для получения графа $\widetilde{G}_{k+1}$ в выделенном пути. Эти способы в совокупности покрывают все возможности применения $O_{1}$ и $O_{2}$, так как отдельные применения операций отличаются только выбором пары вершин для отождествления, а все такие пары вошли в наше рассмотрение.

Мы также выявили три способа применения склейки, которые могут влиять на выполнение условия леммы о смежности $\omega_{j}$ и вершин из $B_{j}^{i}$. Было доказано, что для любого $k$, $i \leqslant k<j$, выполняются неравенства $0 \leqslant \varphi(k)-\varphi(k+1) \leqslant 1$.

Рассмотрим на участке выделенного пути от $\widetilde{G}_{i}$ до $\widetilde{G}_{j}$ три подмножества графов: подмножество $\Theta_{1}$, состоящее из графов, полученных в результате применения операции $O_{1}$ так, как указано в случае 5 в предложении 4, и справедливо равенство $\varphi(k)-\varphi(k+1)=1$; подмножество $\Theta_{2}$, состоящее из графов, полученных в результате применения $O_{2}$, как указано в случае 14 в предложении 5, и справедливо равенство $\varphi(k)-\varphi(k+1)=1$; подмножество $\Theta_{3}$, состоящее из графов, полученных в результате применения $O_{2}$, как указано в случае 15 в предложении 5 , и справедливо равенство $\varphi(k)-\varphi(k+1)=1$.

Для графа $\widetilde{G}_{i}$ выполняется равенство $\varphi(i)=\left|B_{i}\right|$ (согласно предложению 1 , в $\widetilde{G}_{i}$ нет родителей $\left.\omega_{j}\right)$. Для графа $\widetilde{G}_{j}$ равенство $\varphi(j)=0$ выполняется по условию леммы. Следовательно,

$$
\left|B_{i}\right|=\varphi(i)-\varphi(j)=\sum_{i \leqslant k<j}(\varphi(k)-\varphi(k+1)) .
$$


Согласно предложениям 4 и 5 разность $\varphi(k)-\varphi(k+1)$ принимает значение 0 или 1 , причем значение 1 только тогда, когда $\widetilde{G}_{k+1} \in \Theta_{1} \cup \Theta_{2} \cup \Theta_{3}$, значит,

$$
\left|B_{i}\right|=\sum_{\left\{k \mid \widetilde{G}_{k+1} \in \Theta_{1} \cup \Theta_{2} \cup \Theta_{3}\right\}}(\varphi(k)-\varphi(k+1))=\left|\Theta_{1}\right|+\left|\Theta_{2}\right|+\left|\Theta_{3}\right| .
$$

По определению, $\Theta_{1}, \Theta_{2}, \Theta_{3}-$ подмножества графов, соответствующих разным вершинам части выделенного пути длины $j-i$, поэтому справедливо неравенство $\left|\Theta_{1}\right|+\left|\Theta_{2}\right|+\left|\Theta_{3}\right| \leqslant j-i$. Получаем, что $\left|B_{i}\right| \leqslant j-i$. Лемма доказана.

Пусть $\pi\left(K_{p, p}\right)$ - некоторая последовательность сборки графа $K_{p, p}, \Sigma_{\pi}\left(K_{p, p}\right)-$ сеть сборки, соответствующая последовательности сборки $\pi\left(K_{p, p}\right)$, как сказано в определении сети сборки. Пусть граф выделенного пути $\widetilde{G}_{k}, 1 \leqslant k \leqslant \tau$, из сети $\Sigma_{\pi}\left(K_{p, p}\right)$ соответствует графу $G_{s}$ из $\pi\left(K_{p, p}\right)$. Обозначим через $|G|$ число вершин в графе $G$.

Лемма 2. Для любой последовательности $\pi\left(K_{p, p}\right), 1<p$, существует граф $G_{s}$, $1 \leqslant s \leqslant T$, такой, что $p<\left|G_{s}\right|<2 p$ и для любого выделенного пути сети сборки $\Sigma_{\pi}\left(K_{p, p}\right)$, содержащего граф $\widetilde{G}_{k}$, который соответствует $G_{s}$, если $\left|A_{k}\right| \leqslant\left|B_{k}\right|$, то $\left|B_{k}\right|>(1 / 2) p u\left|A_{k}\right|<p$.

Доказательство. Пусть $G_{1}, G_{2}$ и $G_{3}$ - графы некоторой последовательности сборки. Если $G_{3}-$ результат применения $O_{1}$ к паре вершин, одна из которых принадлежит $G_{1}$, другая $-G_{2}$, то

$$
\left|G_{3}\right|=\left|G_{1}\right|+\left|G_{2}\right|-1
$$

Если, допустим, $G_{2}$ - результат применения $O_{2}$ к паре вершин графа $G_{1}$, то $\left|G_{2}\right|=\left|G_{1}\right|-1$.

Для любой последовательности сборки $\pi\left(K_{p, p}\right)$ существует такой минимальный номер $i, 1<i \leqslant T$, что для графа $G_{i}$ из данной последовательности сборки справедливо неравенство $\left|G_{i}\right| \geqslant 2 p$. В противном случае сборка графа $K_{p, p}$ не завершена, так как $\left|K_{p, p}\right|=2 p$.

В последовательности сборки первым, по определению, стоит граф, состоящий из одного ребра и двух вершин, и по условию леммы $p>1$, поэтому необходимо применение некоторых операций для получения $G_{i}$. Поскольку первым в последовательности граф $G_{i}$ имеет $2 p$ или больше вершин, он получен в результате применения $O_{1}$.

Теперь предположим противное, то есть предположим, что не существует графа $G_{S}$ такого, что $p<\left|G_{S}\right|<2 p$. Следовательно, все графы с номером, меньшим $i$, имеют не больше $p$ вершин, так как $G_{i}$ - первый в последовательности имеет число вершин, большее или равное $2 p$. При таком предположении граф $G_{i}$ нельзя получить с помощью $O_{1}$, даже если взять для склейки пару графов с максимально возможным числом вершин $p$. В результате применения операции $O_{1}$ получим граф с числом вершин $p+p-1<2 p$, см. (1). Следовательно предположение неверно, и граф $G_{s}$, удовлетворяющий условию леммы, существует.

Для доказательства второй части утверждения леммы рассмотрим произвольный выделенный путь в сети сборки $\Sigma_{\pi}\left(K_{p, p}\right)$, содержащий граф $\widetilde{G}_{k}$, который соответствует $G_{s}$. Для данного выделенного пути в графе $\widetilde{G}_{k}$ определены подмножества $A_{k}$ и $B_{k}$. Из справедливости неравенств $p<\left|G_{s}\right|<2 p$ следует, что $p<\left|G_{s}\right|=\left|\widetilde{G}_{k}\right|=\left|A_{k}\right|+\left|B_{k}\right|<2 p$. По условию леммы $\left|A_{k}\right| \leqslant\left|B_{k}\right|$ (для определенности). Из системы неравенств $\left|A_{k}\right| \leqslant\left|B_{k}\right|$, $p<\left|A_{k}\right|+\left|B_{k}\right|$ получаем, что $\left|B_{k}\right|>(1 / 2) p$, и из неравенств $\left|A_{k}\right| \leqslant\left|B_{k}\right|,\left|A_{k}\right|+\left|B_{k}\right|<2 p$ получаем, что $\left|A_{k}\right|<p$. Лемма доказана. 
Лемма 3. Для любого $p>1$ верно неравенство $(1 / 2) p<L\left(K_{p, p}\right)$.

Доказательство. Рассмотрим любую последовательность сборки $\pi\left(K_{p, p}\right)$. Данная последовательность заканчивается графом $K_{p, p}$ по определению. Пусть $G_{T} \cong K_{p, p}-$ последний граф последовательности $\pi\left(K_{p, p}\right)$. Рассмотрим произвольный выделенный путь в сети $\Sigma_{\pi}\left(K_{p, p}\right)$. Пусть $\widetilde{G}_{\tau}-$ последний граф данного выделенного пути, следовательно, $\widetilde{G}_{\tau} \cong G_{T} \cong K_{p, p}$. Напомним, что $A_{\tau}$ и $B_{\tau}$, по определению, совпадают с долями полного двудольного графа $\widetilde{G}_{\tau}$.

По лемме 2 в выделенном пути существует граф $\widetilde{G}_{k}$, для которого определены подмножества $A_{k}$ и $B_{k}$, удовлетворяющие условиям $\left|A_{k}\right|<p$ и $\left|B_{k}\right|>(1 / 2) p$.

Как следует из определения вершины-потомка, число потомков вершин любого множества не возрастает при движении к концу выделенного пути. Так как $\left|A_{\tau}\right|=p$ и $\left|A_{k}\right|<p$, получаем, что $\left|A_{\tau}^{k}\right|<\left|A_{\tau}\right|$. Отсюда видно, что существует вершина $\omega_{\tau}$ такая, что $\omega_{\tau} \in A_{\tau}$ и $\omega_{\tau} \notin A_{\tau}^{k}$. Поскольку $\widetilde{G}_{\tau} \cong K_{p, p}$, вершина $\omega_{\tau}$ смежна со всеми вершинами из $B_{\tau}^{k}$. При этих условиях можно применить лемму 1 .

По лемме 1 получаем, что $\tau-k \geqslant\left|B_{k}\right|$. Согласно лемме 2 , как уже говорилось, $\left|B_{k}\right|>(1 / 2) p$. Число вершин в выделенном пути $\tau$ не превосходит, числа вершин в сети сборки $T$. Напомним, что графы выделенного пути нумеруются с единицы, то есть $k \geqslant 1$. В итоге получаем, что $T-1 \geqslant \tau-1 \geqslant \tau-k>(1 / 2) p$. Длина последовательности сборки $\pi\left(K_{p, p}\right)$ равна числу затраченных в ней операций склейки, то есть $T-1$. Сложность сборки $L\left(K_{p, p}\right)$, по определению, равна длине минимальной последовательности сборки. Для любой последовательности сборки графа $K_{p, p}$ длина больше $(1 / 2) p$, поэтому $(1 / 2) p<L\left(K_{p, p}\right)$.

Лемма доказана.

В теореме 1 из [2] были получены верхние оценки сложностей сборки полного двудольного графа и полного графа. Обозначим через $K_{p, q}$ полный двудольный граф с $p$ и $q$ вершинами в соответствующих долях. Данная теорема содержала утверждение о том, что при $q \geqslant p>0$ для $K_{p, q}$ справедливо неравенство $L\left(K_{p, q}\right) \leqslant 3 q+5 \log _{2} q$.

Согласно утверждениям леммы 3 и теоремы 1 статьи [2] для любого $p>1$ выполняются неравенства $(1 / 2) p<L\left(K_{p, p}\right) \leqslant 3 p+5 \log _{2} p$, следовательно, $L\left(K_{p, p}\right) \asymp p$ при $p \rightarrow \infty$.

Теорема 1 доказана.

\section{4. Доказательство теоремы 2}

Доказательство теоремы 2 во многом повторяет доказательство теоремы 1. Следует также отметить, что ситуация заметно упрощается при отсутствии двух долей в графе. Тем не менее, рассмотрим доказательство полностью.

Определения сети сборки и выделенного пути в сети сборки остаются без изменений такими, как в начале доказательства теоремы 1.

Определим по-новому множество вершин $A_{k}, 1 \leqslant k \leqslant \tau$, в графе $\widetilde{G}_{k}$, принадлежащем некоторому выделенному пути в сборке графа $K_{n}$. Теперь $A_{k}$ по определению - просто множество вершин $\widetilde{G}_{k}$.

Пусть $\widetilde{G}_{i}$ и $\widetilde{G}_{j}, i \leqslant j,-$ некоторые графы, принадлежащие выделенному пути. Определение в графе $\widetilde{G}_{j}$ вершины $v_{j}$, являющейся потомком вершины $v_{i} \in \widetilde{G}_{i}$ для данного выделенного пути, остается без изменений таким, как в начале доказательства теоремы 1.

Обозначим через $A_{j}^{i}$ подмножество потомков вершин из $A_{i}$ в множестве $A_{j}$. 
Лемма 4. Для любой сети сборки $\Sigma\left(K_{n}\right)$, для любого выделенного пути в сети сборки $\Sigma\left(K_{n}\right)$, для любой пары графов из данного вылеленного пути $\widetilde{G}_{i} u \widetilde{G}_{j}$, где $1 \leqslant i<j \leqslant \tau$, для любой вериины $\omega_{j}$ такой, что $\omega_{j} \in A_{j} u \omega_{j} \notin A_{j}^{i}$, если $\omega_{j}$ смежна со всеми вершинами из $A_{j}^{i}$, mо $j-i \geqslant\left|A_{i}\right|$.

Доказательство. Рассмотрим произвольную сеть сборки графа $K_{n}$. В данной сети $\Sigma\left(K_{n}\right)$ рассмотрим произвольный выделенный путь. В этом выделенном пути рассмотрим пару графов $\widetilde{G}_{i}$ и $\widetilde{G}_{j}$.

Предложение 6. В графе $\widetilde{G}_{i}$ не сущзествует вериины-родителя $\omega_{i}$, для которой $\omega_{j}$ является потомком.

Доказательство. Поскольку по условию леммы $\omega_{j} \notin A_{j}^{i}$, в графе $\widetilde{G}_{i}$ не существует вершины-родителя $\omega_{i}$, для которой $\omega_{j}$ является потомком. Если бы такая вершина $\omega_{i}$ существовала в $\widetilde{G}_{i}$, то она принадлежала бы $A_{i}$. У любой вершины из $A_{i}$, в частности, у $\omega_{i}$, есть единственный потомок в $\widetilde{G}_{j}$ (по определению вершины-потомка). Этот потомок принадлежит $A_{j}^{i}$ (по определению $A_{j}^{i}$ ), но $\omega_{j} \notin A_{j}^{i}$ по условию леммы 4. Полученное противоречие доказывает предложение 6.

Для предложения 6 также верно следствие 1 о существовании шага $l, i<l \leqslant j$, в выделенном пути, где в графе $\widetilde{G}_{l}$ впервые встречается родитель $\omega_{j}$ при движении от начала к концу.

Определение $R_{k}$ здесь такое же, как в теореме 1 . Также остается в силе предложение 2.

Напомним, что два непересекающихся подмножества вершин графа связаны ребром, если в графе существует хотя бы одно ребро, первый конец которого принадлежит первому подмножеству, второй - второму подмножеству.

Рассмотрим графы $\widetilde{G}_{k}$ и $\widetilde{G}_{k+1}, i \leqslant k<j$. Пусть $\widetilde{G}_{k}^{\prime}$ соответствует ребру с концами $\widetilde{G}_{k}$ и $\widetilde{G}_{k+1}$, лежащему в выделенном пути сети $\Sigma\left(K_{n}\right)$.

Предложение 7. Для любого $k, l-1 \leqslant k \leqslant j-1$, справедливы следуюшие утверждения. Если $R_{k+1}$ и $A_{k+1}^{i}$ связань ребром, то

(a) при получении $\widetilde{G}_{k+1}$ применялась $O_{1}$,

(б) одна из двух вершин для склейки бралась из $A_{k}^{i}$,

(в) не более одной вершины из $A_{k+1}^{i}$ связано ребром с $R_{k+1}$.

Доказательство. Утверждение (а) следует из предложения 2.

Докажем утверждение (б). Пусть $\widehat{G}-$ граф, который был склеен операцией $O_{1}$ с $\widetilde{G}_{k}^{\prime}$ для получения $\widetilde{G}_{k+1}$. Пусть $\hat{v} \in \widehat{G}-$ вершина, выбранная в $\widehat{G}$ для склейки. Рассмотрим следующие два факта. Во-первых, любая вершина в $R_{k+1}$ перешла из $\widehat{G}$, причем без склейки, так как по определению в $R_{k+1}$ нет потомков $\widetilde{G}_{k}$. Во-вторых, $A_{k+1}^{i}$ содержит только потомков вершин из $A_{i}$ по определению. Из этих двух фактов следует, что склейка $\hat{v}$ с любой вершиной из $A_{k} \backslash A_{k}^{i}$ не может привести к связи ребром $R_{k+1}$ и $A_{k+1}^{i}$. Следовательно, существует вершина из $A_{k}^{i}$, которая склеивается с вершиной из $\widehat{G}$. 


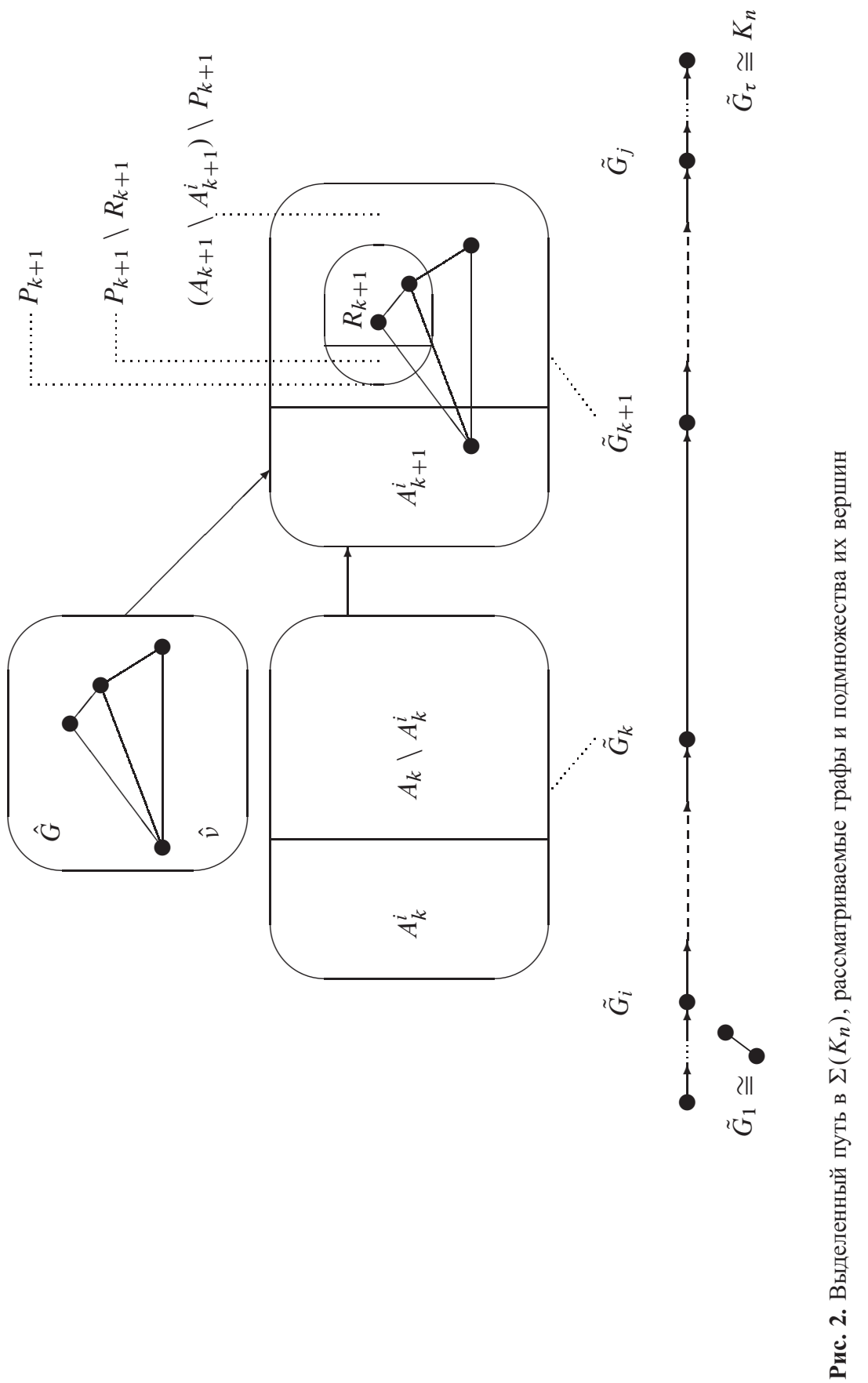


Докажем утверждение (в). Ребра, связывающие $R_{k+1}$ с $A_{k+1}^{i}$, согласно пункту (б), могут появиться только в результате склейки некоторой вершины $\hat{v} \in \hat{G}$ с вершиной из $A_{k}^{i}$. За одну операцию можно склеить только одну вершину из $A_{k}^{i}$ с вершиной $\hat{v}$. Предложение 7 доказано.

Пусть $P_{k}-$ подмножество родителей $\omega_{j}$ в $\widetilde{G}_{k}$. В графе $\widetilde{G}_{k}$ (и соответственно в $\widetilde{G}_{k}^{\prime}$, изоморфном ему) выделим три подмножества вершин: $P_{k},\left(A_{k} \backslash A_{k}^{i}\right) \backslash P_{k}, A_{k}^{i}$. Очевидно, что они не пересекаются, и их объединение равно множеству $A_{k}$ вершин графа $\widetilde{G}_{k}$.

Определим функцию $\varphi(k)$ здесь как число вершин в $A_{k}^{i}$, которые не смежны ни с одной вершиной из $P_{k}$.

Рассмотрим возможности применения операции $O_{1}$ к графу $\widetilde{G}_{k}^{\prime}$. Одна из двух вершин отождествляемой пары должна принадлежать подмножеству из трех перечисленных выше подмножеств $P_{k},\left(A_{k} \backslash A_{k}^{i}\right) \backslash P_{k}, A_{k}^{i}$. Следовательно, есть 3 возможности выбора подмножества путем указания вершины из $\widetilde{G}_{k}^{\prime}$ для склейки $O_{1}$.

Предложение 8. Для любого $k, i \leqslant k<j$, если при получении $\widetilde{G}_{k+1}$ в выделенном пути применяется операция $O_{1}$, то $0 \leqslant \varphi(k)-\varphi(k+1) \leqslant 1$, причем равенство единице возможно только тогда, когда склеиваемая вершина из $\widetilde{G}_{k}^{\prime}$ принадлежит $A_{k}^{i}$.

Доказательство. Пусть $u_{k} \in \widetilde{G}_{k}^{\prime}, i \leqslant k<j$, и $\hat{v} \in \widehat{G}-$ пара вершин, к которым применяется $O_{1}$. Рассмотрим все возможности выбора пары вершин для склейки. Отметим, что при применении $O_{1}$ нас интересуют только возможности выбора вершины из $\widetilde{G}_{k}^{\prime}$. Второй граф $\widehat{G}$, участвующий в склейке, не принадлежит выделенному пути, следовательно, $\widehat{G}$ не имеет своих подмножеств, определяемых через выделенный путь. Поэтому в каждом рассматриваемом случае из $\widehat{G}$ берется произвольная вершина. Перечислим эти возможности.

(1) $u_{k} \in P_{k}$.

(2) $u_{k} \in\left(A_{k} \backslash A_{k}^{i}\right) \backslash P_{k}$.

Для склеек вида 1,2 справедливо равенство $\varphi(k)=\varphi(k+1)$. Действительно, в этих склейках не участвуют вершины из $A_{k}^{i}$, значит, не может измениться их смежность с другими вершинами. Число вершин также не меняется (в частности, не увеличивается, так как все они потомки $A_{i}$ ).

(3) $u_{k} \in A_{k}^{i}$.

Если в результате применения операции $O_{1}$ в графе $\widetilde{G}_{k+1}$ содержатся вершины, у которых нет родителей в $\widetilde{G}_{k}$, то эти вершины содержатся в $A_{k+1} \backslash A_{k+1}^{i}$. Подмножество таких вершин, для которых $\omega_{j}$ является потомком, мы обозначили $R_{k+1}$. По предложению 7 не более одной вершины из $A_{k+1}^{i}$ связано ребрами с $R_{k+1}$, следовательно, в $A_{k+1}^{i}$ по сравнению с $A_{k}^{i}$ добавилось не более одной вершины, смежной с $P_{k+1}$. Другими словами, $\varphi(k)-\varphi(k+1) \leqslant 1$. При операции $O_{1}\left|A_{k}^{i}\right|-\left|A_{k+1}^{i}\right|=0$, и число вершин, смежных с $P_{k}$, не может уменьшиться, значит, $0 \leqslant \varphi(k)-\varphi(k+1)$. Если после применения операции $O_{1}$ подмножество $R_{k+1}=\varnothing$, то не меняется смежность вершин из $A_{k}^{i}$, и также $\left|A_{k}^{i}\right|-\left|A_{k+1}^{i}\right|=0$, значит, $\varphi(k)=\varphi(k+1)$.

Таким образом, только в случае 3 разность $\varphi(k)-\varphi(k+1)$ может быть равной 1 . Предложение 8 доказано. 
Рассмотрим возможности применения операции $O_{2}$ к графу $\widetilde{G}_{k}^{\prime}$ с точки зрения выбора пары вершин для склейки. Каждая вершина из отождествляемой пары может принадлежать одному из трех перечисленных выше подмножеств. Пары отождествляемых вершин не упорядочены, обе вершины в паре могут принадлежать одному подмножеству. Следовательно, есть 6 возможностей выбора пары подмножеств указанием пары вершин для склейки $\mathrm{O}_{2}$.

Предложение 9. Для любого $k, i \leqslant k<j$, если при получении $\widetilde{G}_{k+1}$ в выделенном пути применяется операция $\mathrm{O}_{2}$, то $0 \leqslant \varphi(k)-\varphi(k+1) \leqslant 1$, причем равенство единице возможно только тогда, когда обе склеиваемые вершины принадлежат $A_{k}^{i}$, или одна принадлежит $A_{k}^{i}$, вторая $-A_{k} \backslash A_{k}^{i}$.

Доказательство. Пусть $u_{k}$ и $v_{k}$ - пара вершин графа $\widetilde{G}_{k}^{\prime}, i \leqslant k<j$, к которым применяется $\mathrm{O}_{2}$.

Рассмотрим все 6 возможностей выбора пары вершин для склейки.

(1) $u_{k} \in P_{k}, v_{k} \in A_{k}^{i}$.

(2) $u_{k} \in P_{k}, v_{k} \in\left(A_{k} \backslash A_{k}^{i}\right) \backslash P_{k}$.

Склеек вида 1 и 2 нет. Действительно, $u_{k} \in P_{k}$ только тогда, когда результат склейки $u_{k}$ и $v_{k}$ является родителем $\omega_{j}$ в $\widetilde{G}_{k+1}$. При выполнении этого условия вершина $v_{k}$ также обязана принадлежать $P_{k}$, так как она благодаря данной склейке также является родителем $\omega_{j}$. Однако последнее не выполняется в случаях 1 и 2.

(3) $u_{k}$ и $v_{k}$ принадлежат $\left(A_{k} \backslash A_{k}^{i}\right) \backslash P_{k}$.

(4) $u_{k}$ и $v_{k}$ принадлежат $P_{k}$.

Для склеек вида 3, 4 справедливо равенство $\varphi(k)=\varphi(k+1)$. Это верно, так как в этих склейках не участвуют вершины из $A_{k}^{i}$, значит, не может измениться ни число вершин в $A_{k}^{i}$, ни их смежность с другими вершинами.

(5) $u_{k} \in A_{k}^{i}$ и $v_{k} \in A_{k} \backslash A_{k}^{i}$.

Для операции такого вида $0 \leqslant \varphi(k)-\varphi(k+1) \leqslant 1$. Равенство единице возможно, когда $v_{k}$ смежна с одной или с несколькими вершинами из $P_{k}$. Больше единицы эта разность быть не может потому, что за одну операцию склейки только одна вершина $u_{k} \in A_{k}^{i}$ могла отождествиться с некоторой вершиной из $A_{k} \backslash A_{k}^{i}$.

(6) $u_{k} \in A_{k}^{i}$ и $v_{k} \in A_{k}^{i}$.

Для операции такого вида $0 \leqslant \varphi(k)-\varphi(k+1) \leqslant 1$. Равенство единице возможно, когда хотя бы одна вершина $\left(u_{k}\right.$ или $\left.v_{k}\right)$ не имеет смежной вершины из $P_{k}$. Больше единицы быть эта разность не может потому, что в результате отождествления пары вершин, исчезает не более одной вершины.

Таким образом, только в случаях 5 и $6 \varphi(k)-\varphi(k+1)$ может быть равной 1 . Предложение 9 доказано.

Итак, при рассмотрении трех подмножеств вершин некоторого $\widetilde{G}_{k}, i \leqslant k<j$, возникло 3 способа применения $O_{1}$ и 6 способов применения $O_{2}$ для получения графа $\widetilde{G}_{k+1}$ 
в выделенном пути. Эти способы в совокупности покрывают все возможности применения $O_{1}$ и $O_{2}$, так как отдельные применения операций отличаются только выбором пары вершин для отождествления, а все такие пары вошли в наше рассмотрение.

Мы также выявили три способа применения склейки, которые могут влиять на выполнение условия леммы о смежности $\omega_{j}$ и вершин из $A_{j}^{i}$. Было доказано, что для любого $k$, $i \leqslant k<j$, выполняется неравенство $0 \leqslant \varphi(k)-\varphi(k+1) \leqslant 1$.

Рассмотрим на участке выделенного пути от $\widetilde{G}_{i}$ до $\widetilde{G}_{j}$ три подмножества графов: подмножество $\Theta_{1}$, состоящее из графов, полученных в результате применения операции $O_{1}$ так, как указано в случае 3 в предложения 8, и $\varphi(k)-\varphi(k+1)=1$; подмножество $\Theta_{2}$, состоящее из графов, полученных в результате применения операции $O_{2}$, как указано в случае 5 предложения 9 , и $\varphi(k)-\varphi(k+1)=1$; подмножество $\Theta_{3}$, состоящее из графов, полученных в результате $O_{2}$, как указано в случае 6 предложения 9 , и $\varphi(k)-\varphi(k+1)=1$.

Для графа $\widetilde{G}_{i}$ справедливо равенство $\varphi(i)=\left|A_{i}\right|$ (согласно предложению 6 в $\widetilde{G}_{i}$ нет родителей $\left.\omega_{j}\right)$. Для графа $\widetilde{G}_{j}$ равенство $\varphi(j)=0$ выполняется по условию леммы. Следовательно,

$$
\left|A_{i}\right|=\varphi(i)-\varphi(j)=\sum_{i \leqslant k<j}(\varphi(k)-\varphi(k+1)) .
$$

Согласно предложениям 8 и 9 разность $\varphi(k)-\varphi(k+1)$ принимает значение 0 или 1 , причем 1 только тогда, когда $\widetilde{G}_{k+1} \in \Theta_{1} \cup \Theta_{2} \cup \Theta_{3}$, значит,

$$
\left|A_{i}\right|=\sum_{\left\{k \mid \widetilde{G}_{k+1} \in \Theta_{1} \cup \Theta_{2} \cup \Theta_{3}\right\}}(\varphi(k)-\varphi(k+1))=\left|\Theta_{1}\right|+\left|\Theta_{2}\right|+\left|\Theta_{3}\right| .
$$

По определению, $\Theta_{1}, \Theta_{2}, \Theta_{3}$ - подмножества графов, соответствующих разным вершинам части выделенного пути длиной $j-i$, поэтому $\left|\Theta_{1}\right|+\left|\Theta_{2}\right|+\left|\Theta_{3}\right| \leqslant j-i$. Получаем, что $\left|A_{i}\right| \leqslant j-i$. Лемма доказана.

Пусть $\pi\left(K_{n}\right)$ - некоторая последовательность сборки графа $K_{n}, \Sigma_{\pi}\left(K_{n}\right)-$ сеть сборки, соответствующая последовательности сборки $\pi\left(K_{n}\right)$ по определению сети сборки. Пусть граф выделенного пути $\widetilde{G}_{k}, 1 \leqslant k \leqslant \tau$, из сети $\Sigma_{\pi}\left(K_{n}\right)$ соответствует графу $G_{s}$ из $\pi\left(K_{n}\right)$. Обозначим через $|G|$ число вершин в графе $G$.

Лемма 5. Для любой последовательности $\pi\left(K_{n}\right), 2<n$, существует граф $G_{s}$, $1 \leqslant s \leqslant T$, такой, что $(1 / 2) n<\left|G_{s}\right|<n$ и для любого выделенного пути сети сборки $\Sigma_{\pi}\left(K_{n}\right)$, содержащеге граф $\widetilde{G}_{k}$, который соответствует $G_{s}$, справедливо неравенство $(1 / 2) n<\left|A_{k}\right|<n$.

Доказательство. Напомним следующий факт. Пусть $G_{1}, G_{2}$ и $G_{3}$ - графы некоторой последовательности сборки. Если $G_{3}$ - результат применения $O_{1}$ к паре вершин, одна из которых принадлежит $G_{1}$, другая $-G_{2}$, то

$$
\left|G_{3}\right|=\left|G_{1}\right|+\left|G_{2}\right|-1
$$

Если, допустим, что $G_{2}-$ результат применения $O_{2}$ к паре вершин графа $G_{1}$, то $\left|G_{2}\right|=\left|G_{1}\right|-1$. 
Для любой последовательности сборки $\pi\left(K_{n}\right)$ существует такой минимальный номер $i, 1<i \leqslant T$, что для графа $G_{i}$ из данной последовательности сборки $\left|G_{i}\right| \geqslant n$. В противном случае сборка графа $K_{n}$ не завершена, так как $\left|K_{n}\right|=n$.

В последовательности сборки первым, по определению, стоит граф, состоящий из одного ребра и двух вершин, и по условию леммы $n>2$, поэтому необходимо применение операций для получения $G_{i}$. Граф $G_{i}$ первым в последовательности имеет $n$ или больше вершин, поэтому он получен в результате применения $O_{1}$.

Теперь предположим противное утверждению леммы, то есть предположим, что не существует графа $G_{S}$ такого, что $(1 / 2) n<\left|G_{S}\right|<n$. Следовательно, все графы с номером, меньшим $i$, имеют не больше $(1 / 2) n$ вершин, так как $G_{i}$ первым в последовательности имеет число вершин, большее или равное $n$. При таком предположении граф $G_{i}$ нельзя получить с помощью $O_{1}$, даже если взять для склейки пару графов с максимально возможным числом вершин $(1 / 2) n$. В результате применения операции $O_{1}$ получим граф с $(1 / 2) n+(1 / 2) n-1<n$ вершинами (см. (2)). Следовательно, предположение неверно, и граф $G_{s}$, удовлетворяющий условию леммы, существует.

Для доказательства второй части утверждения леммы рассмотрим произвольный выделенный путь в сети сборки $\Sigma_{\pi}\left(K_{n}\right)$, содержащий граф $\widetilde{G}_{k}$, который соответствует $G_{s}$. Напомним, что $A_{k}$ - множество вершин $\widetilde{G}_{k}$ по определению. С учетом неравенства $(1 / 2) n<\left|G_{s}\right|<n$ находим, что $(1 / 2) n<\left|G_{s}\right|=\left|\widetilde{G}_{k}\right|=\left|A_{k}\right|<n$. Лемма доказана.

Лемма 6. Для любого $n>2$ справедливо неравенство $(1 / 2) n<L\left(K_{n}\right)$.

Доказательство. Пусть $\pi\left(K_{n}\right)$ - произвольная последовательность сборки. Данная последовательность заканчивается графом $K_{n}$ по определению. Пусть $G_{T} \cong K_{n}-$ последний граф последовательности $\pi\left(K_{n}\right)$. Рассмотрим произвольный выделенный путь в сети $\Sigma_{\pi}\left(K_{n}\right)$. Пусть $\widetilde{G}_{\tau}-$ последний граф данного выделенного пути, следовательно, $\widetilde{G}_{\tau} \cong G_{T} \cong K_{n}$. Напомним, что $A_{\tau}$ по определению - множество вершин графа $\widetilde{G}_{\tau}$.

По лемме 5 в выделенном пути есть граф $\widetilde{G}_{k}$, для которого $(1 / 2) n<\left|A_{k}\right|<n$.

Как следует из определения вершины-потомка, число потомков вершин любого множества не возрастает при движении к концу выделенного пути. Так как $\left|A_{\tau}\right|=n$ и $\left|A_{k}\right|<n$ (см. выше), получаем, что $\left|A_{\tau}^{k}\right|<\left|A_{\tau}\right|$. Отсюда видно, что существует вершина $\omega_{\tau}$ такая, что $\omega_{\tau} \in A_{\tau}$ и $\omega_{\tau} \notin A_{\tau}^{k}$. Поскольку $\widetilde{G}_{\tau} \cong K_{n}$, вершина $\omega_{\tau}$ смежна со всеми вершинами из $A_{\tau}^{k}$. При этих условиях можно применить лемму 4.

По лемме 4 получаем, что $\tau-k \geqslant\left|A_{k}\right|$. Согласно лемме 5, как уже говорилось, $\left|A_{k}\right|>(1 / 2) n$. Число вершин в выделенном пути $\tau$ не превосходит, числа вершин в сети сборки $T$. Напомним, что графы выделенного пути нумеруются с единицы, то есть $k \geqslant 1$. В итоге получаем, что $T-1 \geqslant \tau-1 \geqslant \tau-k>(1 / 2) n$. Длина последовательности сборки $\pi\left(K_{n}\right)$ равна числу затраченных в ней операций склейки, то есть $T-1$. Сложность сборки $L\left(K_{n}\right)$, по определению, равна длине минимальной последовательности сборки. Поскольку длина любой последовательности сборки графа $K_{n}$ больше $(1 / 2) n$, справедливо неравенство $(1 / 2) n<L\left(K_{n}\right)$. Лемма доказана.

В теореме 1 из статьи [2] доказано, что $L\left(K_{n}\right)<(7 / 2) n+8 \log _{2} n$ для $K_{n}$. Согласно утверждениям теоремы 1 из [2] и леммы 6 для любого $n>2$ выполняются неравенства $(1 / 2) n<L\left(K_{n}\right)<(7 / 2) n+8 \log _{2} n$, следовательно, $L\left(K_{n}\right) \asymp n$ при $n \rightarrow \infty$. Теорема доказана.

Автор благодарен А. С. Подколзину, под руководством которого выполнена эта работа. 


\section{Список литературы}

1. Яблонский С. В., Введение в дискретную математику. Высшая школа, Москва, 2002.

2. Зайцев Д. В., О сложности сборки графов. Интеллектуальные системы (2005) 9, №1-4, 381-395.

Статья поступила 3.05.2007. 